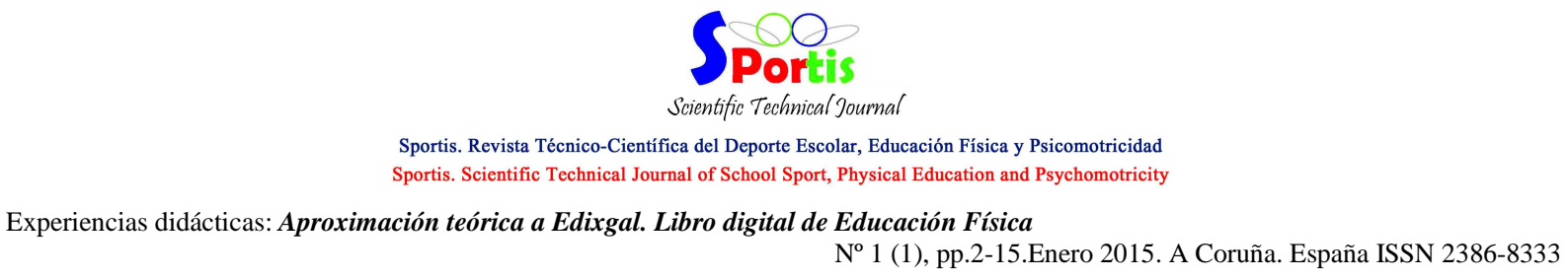

\title{
Aproximación teórica a Edixgal. Libro digital de Educación Física
}

Theoretical approach to Edixgal. Digital Book of Physical Education

\author{
Prof. Dr. Eduardo Rafael Rodríguez Machado (CEIP Raquel Camaño de A Coruña) \\ Email: e.rodriguez.machado@udc.es
}

\section{Resumen}

Uno de los grandes dilemas en el que se encuentra la comunidad educativa es el apostar por libros de texto electrónicos (conocido también como eTextBook) en detrimento de los libros de texto impresos, en Galicia se ha optado en algunos centros ir introduciendo paulatinamente el libro de texto electrónico en las aulas de $5^{\circ}$ Educación Primaria.

En este artículo se concretan las características que definen el libro de texto electrónico y que le hacen resultar atractivo y útil a la comunidad educativa. En primer lugar, se define el libro digital y concretan sus características y los problemas e inconvenientes que nos podemos encontrar con su uso. En segundo lugar, se señala en qué consiste el E-Dixgal y cómo afecta a la introducción de dicho proyecto en el aula. Finalmente, se observa cómo está siendo la introducción del libro digital en relación al área de la Educación Física.

\begin{abstract}
One of the great dilemmas in which the educational community is is the bet on electronic textbooks (aka eTextbook) at the expense of textbooks printed in Galicia has been chosen in some centers to gradually introducing the book electronic text in classrooms 5th Primary Education.

This article describes the characteristics that define the electronic textbook and make you be attractive and useful to the educational community are realized. First, it defines the digital book and materialize their characteristics and problems and drawbacks that we can find to use. Secondly, it states what the E-Dixgal and how it affects the introduction of the project in the classroom. Finally, we see how being the introduction of digital books in relation to the area of Physical Education
\end{abstract}

Palabras clave: libro de texto electrónico, plataforma virtuales digitales, educación física, TICs, E-Dixgal.

Keywords: electronic textbook, digital virtual platform, physical education, ICT, E-Dixgal. 


\section{Introducción}

$\mathrm{N}^{\circ} 1$ (1), pp.2-15.Enero 2015. A Coruña. España ISSN 2386-8333

El proyecto Educación Digital (E-DIXGAL) tiene como objeto favorecer la incorporación generalizada de las tecnologías de la información y de la comunicación (TIC) en el desarrollo de la actividad educativa, facilitando a los centros adscritos a la red Abalar la disponibilidad de libros y otros materiales digitales con los que puedan desarrollar su proyecto en la totalidad del currículo de $5^{\circ}$ de Educación Primaria en el curso 2014-2015, ampliándose a otros cursos y etapas en los años siguientes.

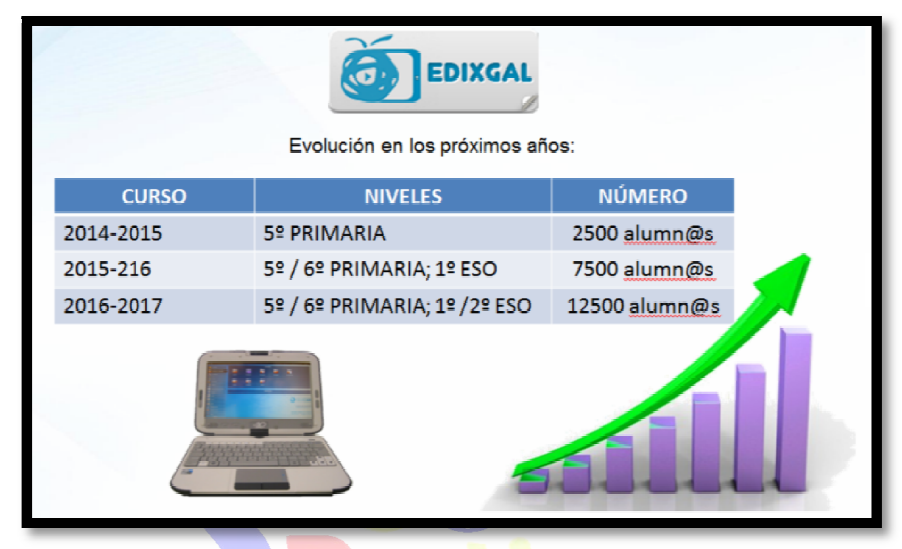

Imagen $n^{\circ} 1$. Evolución de Edixgal en Galicia.

El proyecto E-DIXGAL que está en funcionamiento en este curso 2014/2015 incorporando a 2.500 alumnos de $5^{\circ}$ de primaria. En el curso 2015/16 la previsión es que la plataforma esté disponible en $5^{\mathrm{a}}$ y $6^{\mathrm{a}}$ de primario y $1^{\mathrm{o}}$ de ESO con un alcance de 7.500 alumnos y que en el tercer año del proyecto, en el curso 2016/2017 sea empleado por 12.500 alumnos de $5^{\circ}$ y $6^{\circ}$ de Primario y $1^{\circ}$ y $2^{\circ}$ de ESO. Así, en 3 años el proyecto llegará a un total de 22.500 estudiantes. En la plataforma de aprendizaje virtual (EVA-Abalar), los alumnos acceden tanto de manera on-line como off-line a contenidos curriculares completos y gratuitos para las familias facilitadas por la Xunta como a aquellos elaborados por los propios docentes de los centros educativos. Además, esta nueva herramienta facilita el acceso a los contenidos de pago de empresas editor gracias a la conexión con el Punto Neutro del Ministerio de Educación.

El CEIP Raquel Camacho (A Coruña) apuesta por la integración de las Tecnologías de la Información y Comunicación (TIC), lo que supone para el profesorado el reto de innovar sus prácticas docentes y además un cambio de las infraestructuras físicas y organizativas del centro. 
En nuestro centro utilizamos dentro del Aula Abalar diferentes herramientas web, el servidor del centro, herramientas de autor, aplicaciones de Realidad Aumentada, la PDI, la red Edmodo y ahora una nueva herramienta el libro digital.

\section{Breve descripción del contexto del a experiencia.}

El Proyecto Abalar se manifiesta en el uso personalizado de un ordenador portátil por parte del alumnado de $5^{\circ}$ y $6^{\circ}$ de primaria y $1^{\circ}$ y $2^{\circ}$ de la ESO acompañada de la puesta en marcha de lo que se ha dado en llamar las aulas digitales del siglo XXI, es decir, aulas dotadas de una adecuada infraestructura tecnológica y de conectividad por banda ancha.

Este ambicioso programa, se articula en cinco ejes de intervención:

- dotación de aulas digitales,

- desarrollo de la conectividad a Internet desde el centro educativo y desde los hogares e interconectividad dentro del aula,

- formación del profesorado,

- generación y acceso a contenidos educativos digitales de carácter curricular,

- e implicación de los alumnos y sus familias en el uso y aprovechamiento de estos recursos.

La estrategia del proyecto Abalar gira en torno al concepto de centro educativo digital. El centro educativo digital supera el concepto del centro educativo tradicional y persigue disponer de recursos humanos y pedagógicos, así como de infraestructuras y servicios adecuados para el uso cotidiano de la tecnología por parte de todos los agentes de la comunidad educativa, en la actividad docente, de aprendizaje y administrativa.

Nuestras 4 aulas Abalar cuentan con un miniportátil para cada alumno con un armario de carga, una PDI y cañón de última generación y un ordenador del profesor, todos estos elementos están comunicados por wifi-Abalar y el servidor del centro. 


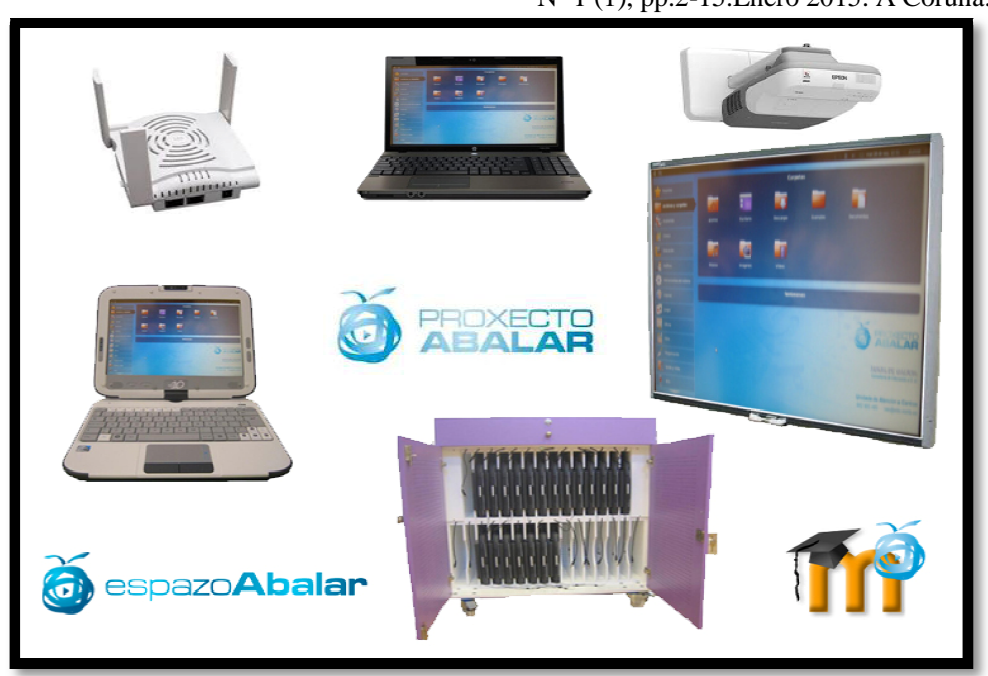

Figura $\mathrm{n}^{\circ}$ 2. Elementos del aula Abalar

En estos momentos estamos participando en el proyecto de carácter experimental de EDIXGAL ( 84 centros en toda Galicia), una plataforma de aprendizaje virtual en la cual los alumn@s acceden a contenidos curriculares "completos y gratuitos" facilitados por la Xunta, así como a aquellos elaborados por los propios docentes en los centros educativos.

\section{Objetivos}

El objetivo principal es determinar si cabe apostar por el libro de texto electrónico, puliendo los defectos actuales, o bien rechazar esta posibilidad y optar por otro tipo de propuestas basadas en las TIC que aporten beneficio a la comunidad educativa.

Para alcanzar el objetivo principal, se plantea la siguiente ruta de investigación:

- Primer paso: Definir el libro de texto electrónico y concretar sus características.

- Segundo paso: Problemas e inconvenientes del uso del libro de texto electrónico.

- Tercer paso: Hacer un análisis de la plataforma virtual que se utiliza en E-DIXGAL.

- Cuarto: Hacer un análisis del libro de Educación Física para $5^{\circ}$ Educación Primaria dentro del programa.

\section{Evolución}

Ya en los años 70, analizando los libros de texto (Domènechs, 1992), se llegaba a la conclusión que éstos no eran más que un compendio de contenidos cerrados que determinaba un modelo primordialmente expositivo. 
Con la llegada de las nuevas tecnologías, los educadores ven una oportunidad de modificar esta concepción del libro de texto. Esta adopción de la tecnología en las clases se lleva a cabo de manera gradual, por etapas (Dwyer, 1991):

- Primero tratan de replicar los recursos y estrategias tradicionales usando la tecnología existente. Por ejemplo, digitalizando un texto en formato PDF (Portable Document Format, de la compañía Adobe).

- Después intentan modificar esos recursos y estrategias para dotarlos de un valor añadido. Por ejemplo, añadir material multimedia al texto digitalizado en formato PDF.

- Y finalmente, algunos educadores transforman el proceso de enseñanza-aprendizaje de tal manera que la adopción de las nuevas tecnologías resulta imprescindible.

Para poder definir correctamente lo que se entiende por libro de texto electrónico, se debe pensar en la transformación del proceso de enseñanza (tercera etapa) ya que limitarse a las dos primeras etapas significa desvirtuar el concepto de libro de texto electrónico.

\section{Libro de texto electrónico}

A continuación, se muestran algunas definiciones de libro de texto electrónico de diversos autores:

- Son versiones digitales de libros impresos, que se distribuyen a través de internet. Estos archivos pueden ser leídos en lectores electrónicos, tabletas, ordenadores personales, teléfonos inteligentes y también en algunos móviles (Coopers, 2011).

- Un libro electrónico presenta el texto e información gráfica en formato digital, y por lo general utiliza la navegación interactiva para facilitar el movimiento entre los diferentes capítulos y páginas (Davison et al., 2005).

- Dispositivo que debe ser capaz no solo de ajustar el texto en la pantalla, si no también debe mostrar vídeo, audio y contenido multimedia, así como también tener capacidad de computación y soporte de entradas y salidas. Además se debe apoyar en el uso de estándares y ser capaz de reunir los datos de la interacción del usuario (González Crespo et al., 2010).

Se puede observar cierta ambigüedad al usar el término libro electrónico: para referirse al archivo con el contenido digital (eBook o eTextBook) o para el dispositivo que proporciona la visualización e interacción con dicho contenido (eReader, Intelligent eReader o Tablet).

Cronograma editorial:Artículo recibido: 11/12/2014 Aceptado: 28/12/2014 Publicado: 10/01/2015

Para citar este artículo utilice la siguiente referencia: Rodríguez, E.R. (2015). Aproximación teórica a Edixgal. Libro digital de Educación Física. SportisScientificTechnicalJournal, 1 (1), 2-15. 
Del análisis de estas definiciones se deduce que entre el dispositivo y el usuario debe haber ciertos mecanismos de interacción como pueden ser la pantalla táctil, el teclado virtual inteligente, sistemas de reconocimiento de voz, orientación automática de la pantalla...

Los mecanismos de interacción facilitan que cualquier persona pueda utilizar un dispositivo de manera sencilla, amigable y eficiente.

Otro aspecto a concretar debe ser el formato de los archivos electrónicos. En los últimos años, los formatos más comúnmente utilizados han sido el ePub (Electronic Publication) y el PDF (Adobe Portable Document). Son formatos estáticos, orientados hacia la lectura en dispositivos portátiles, que pueden incluir contenido multimedia pero que carecen de una interacción más profunda con el usuario. Es por tanto necesario ir un poco más allá de estos formatos tradicionales y estudiar otras opciones.

- Microsoft Reader (archivos .lit): El Microsoft Reader utiliza una tecnología que hace posible una navegación mediante teclado, ratón o lápiz óptico en los archivos .lit de tal forma que el usuario puede añadir anotaciones, resaltados y marcadores en cualquier página del texto o crear dibujos de forma libre en las páginas del lector.

Apple iBooks 2 (archivos .ibook): En el 2012, Apple renueva su aplicación para la lectura de libros en formato ibook en los que pueden integrar fotos, vídeos y animaciones interactivas. Al igual que en el Microsoft Reader se puede subrayar, añadir notas, realizar búsquedas,... Pero además incluye algunas herramientas dirigidas a la comunidad educativa que permiten, por ejemplo, realizar test interactivos auto-corregibles.

A modo de resumen, se pretende que el libro de texto electrónico no sea un soporte únicamente de lectura, sino que además incluya contenido multimedia manipulable fácilmente por el usuario y que éste a su vez sea capaz de interactuar con el dispositivo, ya sea subrayando, anotando, buscando palabras,... de forma fluida y eficiente.

\section{Problemas e inconvenientes del uso del libro de texto electrónico}

A continuación se revisan los principales problemas e inconvenientes que se derivan de la adopción del libro de texto electrónico en substitución del formato tradicional impreso.

\section{La tablet digital o el netbook (miniportátil)}

La introducción de la tablet digital o el netbook en el aula para su manipulación por menores implica una educación previa, no tanto en su funcionamiento (nativos digitales), pero sí en su Cronograma editorial:Artículo recibido: 11/12/2014 Aceptado: 28/12/2014 Publicado: 10/01/2015 
$\mathrm{N}^{\mathrm{o}} 1$ (1), pp.2-15.Enero 2015. A Coruña. España ISSN 2386-8333 cuidado: son aparatos delicados, sensibles y caros de reemplazar. Conviene reservarles un espacio protegido dentro del aula con acceso a recarga eléctrica cuando no están en uso. Utilizar fundas protectoras robustas y protectores de pantalla para evitar golpes y rayaduras será de vital importancia.El acceso a internet o a un servidor para comunicarse con otras tabletas y/o el ordenador del profesor debería de estar gestionado adecuadamente para evitar colapsos en el ancho de banda y proteger al alumnado de contenidos inadecuados.

\section{Sindrome de visión por computador}

El uso prolongado frente a una tablet o miniportátil puede llevar a una serie de problemas relacionados con la visión: cansancio de la vista, dolores de cabeza, visión borrosa, ojos secos, dolor de cuello y hombro,...

Por ello, expertos como (Kozeis, 2009) sugieren una serie de puntos a tener en cuenta para niños que utilizan un ordenador durante largos períodos:

- Un examen de ojos anual. Esto nos asegura que el niño puede ver clara y cómodamente.

- Reducción de la cantidad de tiempo que el niño puede utilizar continuamente el ordenador. Un descanso de diez minutos por cada hora de trabajo reducirá al mínimo el desarrollo de problemas de acomodación y la irritación de los ojos.

- Reducción de la cantidad de luz en la habitación. En algunos casos, se prefiere una luz más tenue en lugar de la luz del techo brillante.

En definitiva, un esfuerzo a la hora de tomar precauciones puede ayudar a reforzar los hábitos de visualización apropiados y garantizar un uso cómodo y agradable de las tablets o miniportátiles.

A nivel educativo

Se deben tener en cuenta ciertos inconvenientes:

- Escritura. Una de las habilidades que, junto con la lectura y la expresión, un alumno de primaria debe aprender es la escritura. Las tablets o miniportatiles no están diseñadas para la escritura. Podemos utilizar teclados virtuales o físicos, lápices ópticos, los dedos,... Pero es evidente que comunicarnos con el dispositivo no es lo mismo que adquirir una caligrafía, una correlación mano-ojo, en definitiva, un "saber escribir". Es por este motivo que la adquisición de esta habilidad no puede ni debe 


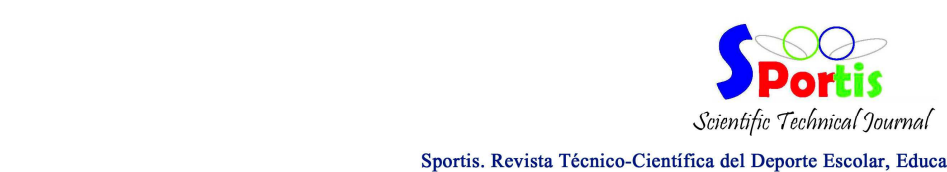

Sportis. Revista Técnico-Científica del Deporte Escolar, Educación Física y Psicomotricidad

Sportis. Scientific Technical Journal of School Sport, Physical Education and Psychomotricity

Experiencias didácticas: Aproximación teórica a Edixgal. Libro digital de Educación Física

$\mathrm{N}^{\mathrm{o}} 1$ (1), pp.2-15.Enero 2015. A Coruña. España ISSN 2386-8333 hacerse a través de una tableta digital. Por lo tanto, el uso de tablets o miniportatiles debe complementarse con el uso de cuadernos o libretas y lápices.

- Trabajo creativo. Aunque cada vez sea mayor el número de aplicaciones interactivas que nos ofrece el mercado, las tablets se han diseñado principalmente para el consumo de contenidos: correo electrónico, redes sociales, videos,... Tareas de creación como editar textos, implemetar tablas de datos o realizar presentaciones siempre será mucho más fácil en un ordenador de sobremesa o en un portátil. Es por ello que las tablets digitales deben considerarse principalmente como una herramienta de adquisición de contenidos, con posibilidad de cierta interacción, pero que necesita complementarse con otros medios para que el alumno desarrolle la totalidad de las habilidades consideradas en el currículum de primaria.

- Falta de formación. El profesorado, immigrante digital en el mejor de los casos, que ha aprendido a utilizar presentaciones, pizarras digitales, programas de registro de notas,... Nuevamente debe adoptar una nueva tecnología e introducirla en el aula. Lo más probable es que opte por copiar recursos y estrategias tradicionales (Dwyer, 1991), adaptando la nueva tecnología a la forma habitual de enseñar. Por lo tanto, es imprescindible formar al profesorado para que desarrollen nuevos medios de enseñar a sus alumnos y que éstos creen sus propios artefactos de aprendizaje.

\section{La propuesta de editoriales en Edixgal}

En el negocio emergente del libro de texto electrónico, podemos hacer una distinción de tres tipos de editoriales:

- Editoriales tradicionales. Como el nombre indica, son las editoriales que hasta hace relativamente poco tiempo tenían como único producto el libro de texto tradicional y que ahora se han visto obligadas a introducir el libro de texto electrónico en su catálogo.

- Editoriales de mercado. Este grupo de editoriales ha ido más allá y ha creado un mercado específico para la venta de sus libros electrónicos a través de una aplicación o de un lector (Reader).

- Editoriales de plataforma. Finalmente tenemos el grupo que más ha apostado por el libro electrónico, creando sus propios lectores y aplicaciones en los que se pueden

Cronograma editorial:Artículo recibido: 11/12/2014 Aceptado: 28/12/2014 Publicado: 10/01/2015 


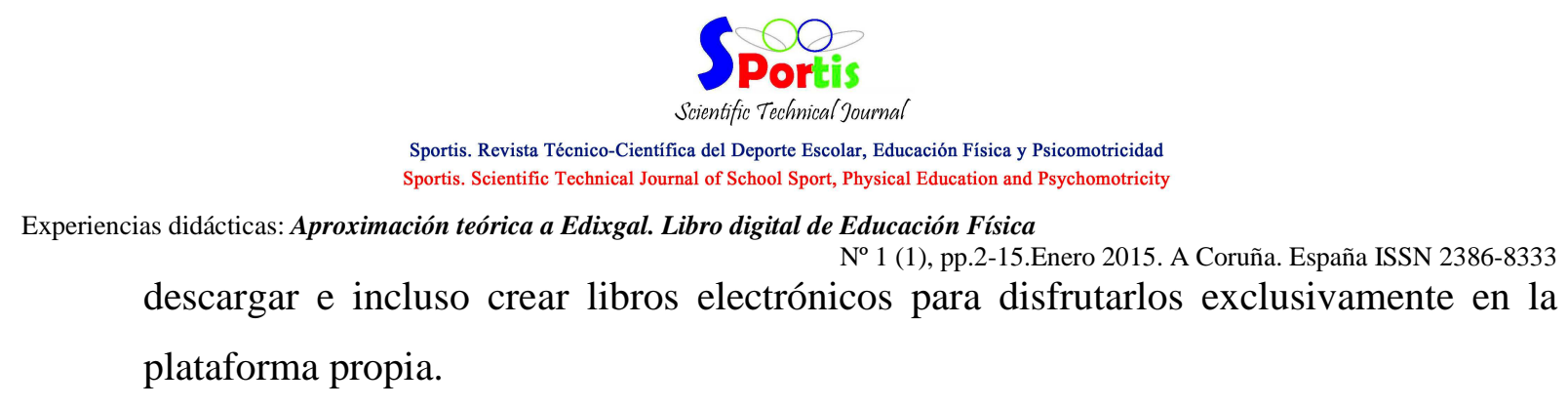

Esta última es por la que se ha decantado el programa EDIXGAL, los docentes y alumnos de los centros implicados desarrollan su actividad educativa diaria en un entorno virtual de aprendizaje que les permite acceder a contenidos digitales para $5^{\circ}$ de primaria de dos proveedores, la editorial EDEBÉ y la empresa de contenidos digitales Netex.

Los docentes podrán elaborar su propia "mochila digital" seleccionando a través de la plataforma digital los contenidos formativos y pudiendo incorporar los materiales complementarios que se deseen, así como los contenidos de elaboración propia. Cabe recordar que estos centros, integrados en el proyecto Abalar, tienen también a su disposición el espazoAbalar con más de 2.155 recursos educativos digitales complementarios en siete idiomas.Los alumnos acceden al material seleccionado por el profesor tanto a través de una conexión a internet (modo online) como desde su netbook (modo offline). De esta forma, se garantiza que los alumnos y las alumnas que no dispongan de conexión a internet desde sus casas puedan seguir el curso sin dificultad. De hecho, todo avance que realicen sin conexión será actualizado de manera automática en la plataforma en cuanto el netbook vuelva a tener conexión de red.

\section{La plataforma virtual: EVA Abalar}

Para el desarrollo del proyecto se creó una plataforma virtual en Moodle 2.6. El acceso a la plataforma virtual de aprendiza es posible a través de cualquier equipo con conexión a internet siempre que se haga uso de las claves de acceso que les serán entregadas al alumnado en el centro. De este modo el alumno que así lo desee, tienen la posibilidad de utilizar también en su casa el netbook que se utiliza en la clase, para lo que deberá solicitar en su centro un formulario de préstamo del mismo, que devolverá firmado en el plazo más breve posible.La utilización del ordenador portátil y de las herramientas y contenidos digitales instalado en el mismo son gratuitos para el alumno. Las familias se comprometen a que el alumnado realice un uso diligente del equipamiento, al cuidado de un equipo público, que podrá ser usado en otros cursos por otros alumnos, y a una utilización vinculada a la actividad educativa para la que es cedido.

La URL para acceder a la plataforma EVA Abalares: http://eva.edu.xunta.es

\footnotetext{
Cronograma editorial:Artículo recibido: 11/12/2014 Aceptado: 28/12/2014 Publicado: 10/01/2015 
Experiencias didácticas: Aproximación teórica a Edixgal. Libro digital de Educación Física

$\mathrm{N}^{\circ} 1$ (1), pp.2-15.Enero 2015. A Coruña. España ISSN 2386-8333

En la pantalla de acceso a EVA Abalar, es por donde se realiza la identificación en la plataforma.

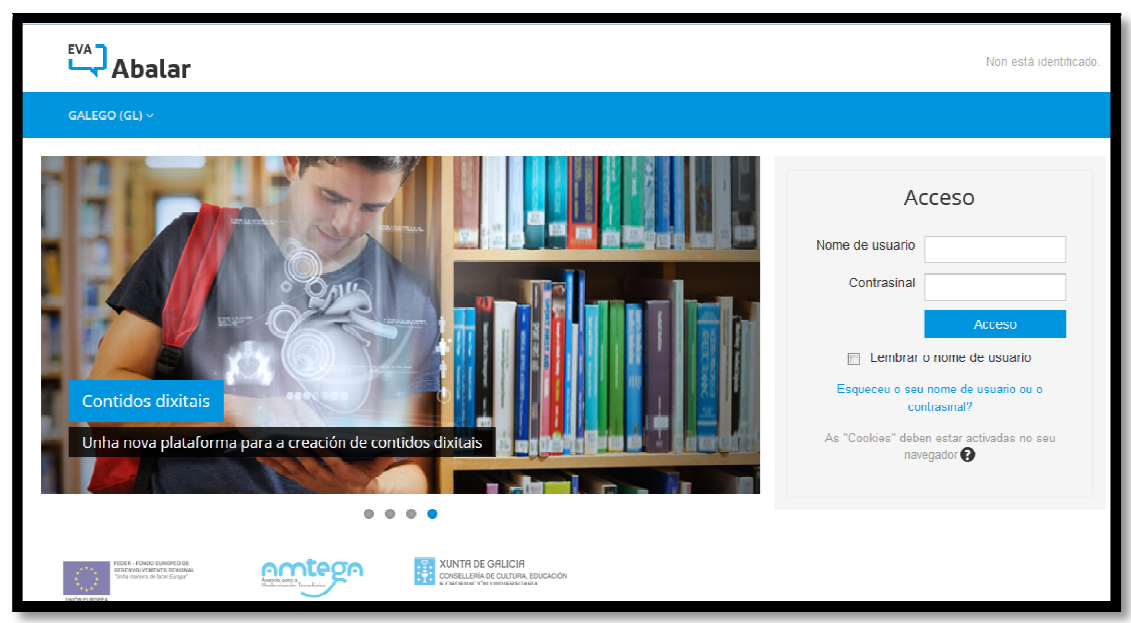

Imagen $\mathrm{n}^{\mathbf{0}}$ 3. Pantallazo de la plataforma EVA Abalar

Para solucionar incidencias técnicas en el uso de entorno virtual de aprendizaje, tanto los profesores como las familias contarán con un equipo de soporte técnico (Amtega) y también de la red social del profesorado (Redeiras), donde existe un grupo de coordinadores de EDixgal para solucionar aquellos problemas que vayan surgiendo.

Dentro de la plataforma se encuentran alojados los dos libros digitales puestos a disposición por parte de la Consellería. Por una parte tenemos el libro de Edebé que es un pdf enriquecido y por otro lado la empresa Netex se ha encargado de elaborar contenidos e-learning HTML5 para cada una de las materias de $5^{\circ}$ de Educación Primaria.

\section{El libro de Educación Física para $5^{\circ}$ Educación Primaria}

También disponemos dentro de la plataforma el libro digital de la materia de Educación Física tanto en la editorial Edebé como en la plataforma digital Netex.Una vez que accedemos a nuestra área personal debemos matricular a nuestros alumnos para que ellos puedan acceder al contenido de Educación Física.

El docente debe hacer el proceso una sola vez al inicio de cada curso escolar en cada una de las asignaturas que tutoriza, o cada vez que cree un curso y desee que los alumnos puedan automatricularse. 


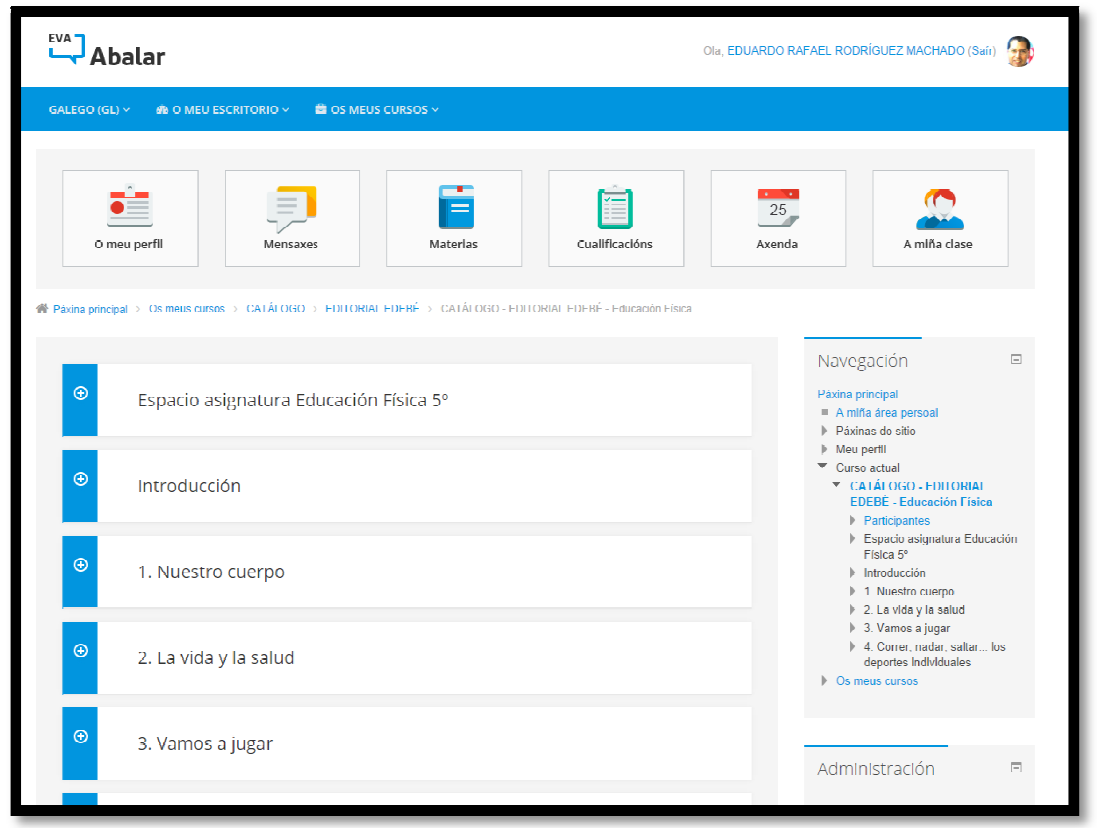

Imagen $n^{\circ}$ 4. Plataforma de EVA Abalar-Educación Física

En el catálogo de Edebé de la materia de Educación Física de $5^{\circ}$ Primaria podemos acceder a los contenidos publicados por la editorial y a los que podemos añadir nosotros como docentes.

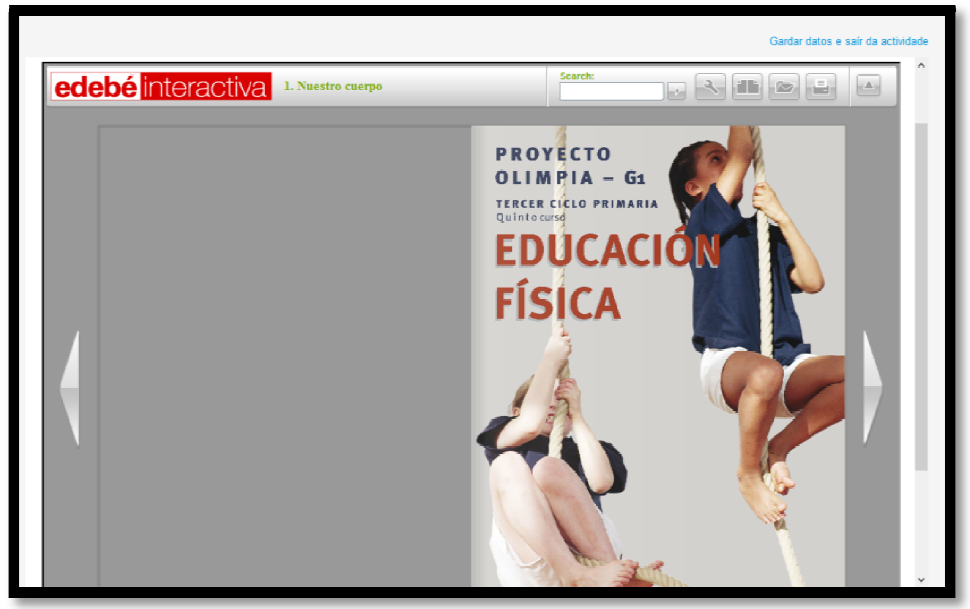

Imagen nº 5. Plataforma Edebé-Educación Física

Serían los siguientes: 1. Introducción,2. Nuestro cuerpo, 3. La vida y la salud, 4. Vamos a jugar, Correr, nadar, saltar... los deportes individuales 
En el aula virtual de Netexelearning podemos acceder al contenido tanto para el alumno como el profesor.

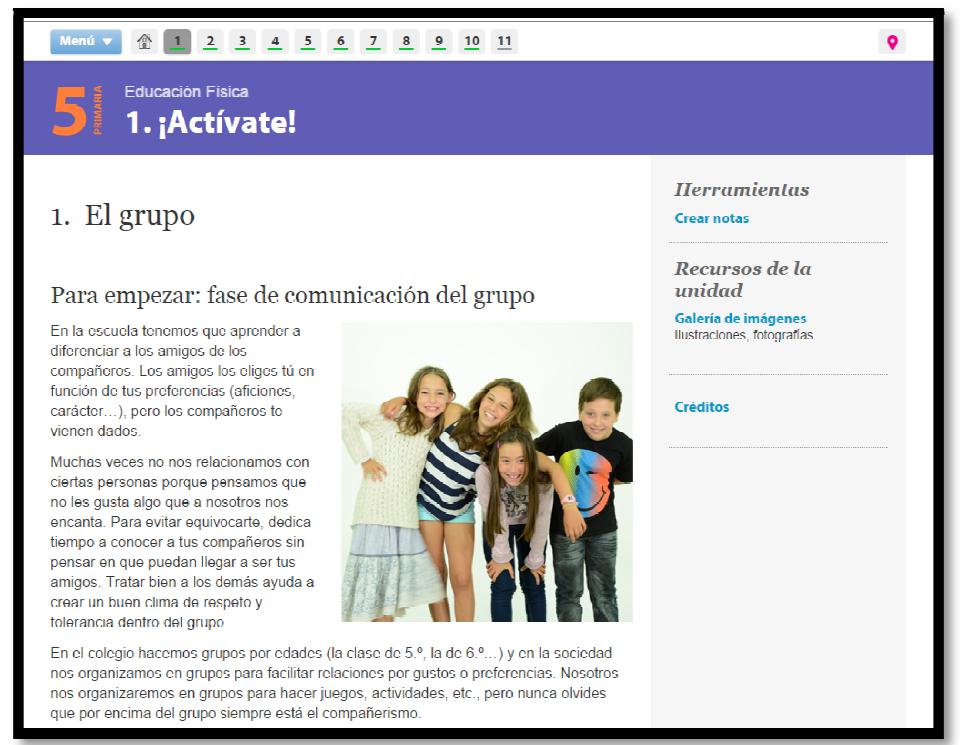

Imagen $n^{\circ}$ 6. Plataforma Netex-Educación Física

En el tenemos las siguientes unidades:1. ¡Actívate!, 2. ¡Empieza el espectáculo!, 3. ¡Toca la banda!, 4. ¿Quién es quién?, 5. Minideportes, 6. Jugando en la naturaleza.

EVA Offline permite acceder a los libros digitales sin conectividad una vez que estos han sido descargados.

\section{Conclusiones}

Se ha definido el concepto de libro electrónico de tal forma que la adopción de éste debe suponer un cambio en el proceso de enseñanza-aprendizaje y no una copia de recursos y estrategias tradicionales usando la tecnología existente.El profesorado valora la puesta en marcha de E-Dixgal, aunque reconocen los posibles inconvenientes de una enseñanza íntegramente digital. Los expertos señalan que la utilización del ordenadores y pantallas en vez de libros de texto es un mero cambio de soporte, por lo que la clave de una buena educación "no está en el instrumento en sî", sino en la transmisión del profesorado y en el modo de trabajar los contenidos. 
Los docentes recuerdan que buena parte del alumnado, nativo digitales, aventajan a los mayores en el manejo de las nuevas tecnologías. Pese a ello, consideran fundamental que "aprendan a diferenciar contenidos importantes de los que no lo son, y los que tienen validez de los que no las tienen". En ese sentido, coinciden con los expertos en TIC en que los docentes deben estar preparados para impartir los contenidos con las nuevas herramientas, un aprendizaje al que se compromete la Consellería de Educación. Ambos sectores sostienen que el nuevo programa sin libros de texto permitirá una reducción importante en el gasto de las familias, ya que el material y los contenidos educativos se impartirán de forma gratuita.

Una consecuencia de introducir el libro de texto electrónico en el aula es que ayuda a potenciar la enseñanza guiada por el profesor, pasando a un modelo personalizado para cada estudiante, haciéndolos más protagonistas y participativos en el proceso de su aprendizaje. La autocorrección de ejercicios, la búsqueda de palabras en el glosario, resaltar o anotar, o activar figuras tridimensionales, hace que el proceso de aprendizaje ya no sea lineal sino que se adapte a las capacidades de los alumnos: algunos necesitarán repetir los ejercicios, otros repasar palabras clave del glosario, otros ejercitar su capacidad espacial mediante figuras tridimensionales, ...

Creemos que debemos ser precavidos ante la implantación del programa, recordando que no debe olvidarse el "perfeccionamiento de la escritura sobre el papel, además de centrarse en las destrezas básicas, en la parte de contenidos. Los niños y niñas deben de escribir correctamente, una habiliddad que se consigue "practicando la escritura y estudiando normas de ortografía", por lo que el esfuerzo debe ir más allá de "saber utilizar las pantallas".

Con respecto a la materia de Educación Física los contenidos que disponemos en la plataforma son aún muy pobres, el profesor debe utilizar la plataforma y aprovechar los recursos que nos ofrece la red y moodle para ir completando su aula elaborando tareas, cuestionarios, wiki, pruebas,... y utlizando los herramientas de que dispone en la plataforma. Proyectos como Moodle, una comunidad de aprendizaje en línea que apuesta por un aprendizaje colaborativo y centrado en el alumno, serán los que a partir de ahora comiencen a sonar como nuevas formas de generar y gestionar recursos educativos en el aula.

La comunidad educativa cada vez tiene menos excusas para adoptar las nuevas tecnologías que le permitirán transformar el proceso de enseñanza-aprendizaje, situando al alumno como principal protagonista y dejando al profesor como guía. 


\section{Referencias bibliográficas}

$\mathrm{N}^{\mathrm{o}} 1$ (1), pp.2-15.Enero 2015. A Coruña. España ISSN 2386-8333

1. Domènechs, J y Viñas, J, (1992). Movimientos de Renovación Pedagógica. Cuadernos de Pedagogía 199, 12-18

2. Dwyer, D. C., Ringstaff, C., \& Sandholtz, J. H. (1991). Changes in teachers' beliefs and practices in technology-rich classrooms. Educational Leadership, 48(8), 45-52.

3. Coopers, P. (2011). Turning the page: The future of ebooks. Recuperado 10-09-2014) http://www.pwc.com/en_GX/gx/entertainment-media/pdf/eBooks-TrendsDevelopments.pdf.

4. Consellería de Educación Xunta de Galicia. Repositorio Abalar. Recuperado (03-092014) http://www.edu.xunta.es/espazoAbalar/espazos/recursos

5. Consellería de Educación Xunta de Galicia. EDixgal. Recuperado (09-09-2014) http://www.edu.xunta.es/espazoAbalar/es/noticia/e-dixgal-implantacion-del-librodigital-en-los-centros-educativos

6. Davison, G., Murphy, S, and Wong, R. (2005). The use of ebooks and interactive multimedia as alternative forms of technical documentation. In Proceedings of the 23rd annual international conference on Design of communication: documenting \& designing for pervasive information, SIGDOC ' $05, \mathrm{NY}$, USA.

7. González Crespo, R., Sanjuán Martínez, O., Cueva Lovelle, J. M., Pelayo GarcíaBustelo, B. B., Gayo, J. E. L. (2010). Recommendation system based on user interaction data applied to intelligent electronic books. Computers in Human Behavior.

8. Kozeis, N (2009). Impact of computers use on children's vision. Revista Hippokratia, 2009 Oct-Dec; 13(4): 230-231. Recuperado http://www.ncbi.nlm.nih.gov/pmc/articles/PMC2776336/.

9. De Puelles Benítez, M. (2007). La política escolar del libro de texto en la España contemporánea. Avances en Supervisión Educativa, $\mathrm{n}^{\circ}$ 6. Recuperado (05-092014)http://www.adide.org/revista/index.php?option=com_content\&task=view\&id=1 98\&Itemid $=47$. 